\title{
Cosmological magnetic field and dark energy as two sides of the same coin
}

\author{
Ariel R. Zhitnitsky \\ Department of Physics and Astronomy, University of British Columbia, \\ Vancouver, British Columbia V6T 1Z1, Canada
}

(Received 7 March 2019; published 16 May 2019)

\begin{abstract}
It has been recently argued [Phys. Rev. D 98, 045008 (2018)] that the de Sitter phase in cosmology might be naturally generated as a result of the dynamics of the topologically nontrivial sectors in a strongly coupled QCD-like gauge theory in the expanding Universe. It is known that the de Sitter phase is realized in the history of our Universe twice: The first occurrence is coined as inflation, while the second time (which is occurring now) is dubbed as dark energy (DE). The crucial element of the proposal is the presence of a nontrivial gauge holonomy which is the source of the vacuum energy leading to the de Sitter behavior. It has been also argued that the anomalous coupling of the system with the Standard Model particles leads to the reheating epoch in the case of the inflationary phase. A similar anomalous coupling of the system with the Maxwell electromagnetic field during the DE epoch generates the cosmological magnetic field. The intensity of the field is estimated on the level of $10^{-10} \mathrm{G}$, while the corresponding correlation length reaches the scale of the visible Universe.
\end{abstract}

DOI: 10.1103/PhysRevD.99.103518

\section{INTRODUCTION}

This work is mostly motivated by a recent proposal [1] where vacuum energy (and accompanying it the de Sitter phase) is dynamically generated. The proposal [1] can be viewed as a synthesis of two naively unrelated ideas discussed previously in Refs. [2-4] and [5-7], correspondingly. On the gravity side [2-4], the nontrivial element of the construction is represented by the Euclidean spacetime with a time compactified to a circle $\mathbb{S}^{1}$. On the gauge field theory side [5-7], the same $\mathbb{S}^{1}$ plays a crucial role when the gauge configurations may assume a nontrivial holonomy along $\mathbb{S}^{1}$. Precisely, the gauge configurations with the nontrivial holonomy along $\mathbb{S}^{1}$ may serve as a source of vacuum energy density, which eventually leads to the de Sitter behavior.

The focus of the proposal [1] coined as "holonomy inflation" was the study of vacuum energy and the corresponding de Sitter behavior in an application to the inflationary Universe. It has been also suggested in that proposal that the holonomy inflation ends as a result of anomalous coupling of the system with massless Standard Model (SM) gauge fields with known coefficients.

The present work applies the same ideas on the dynamical generation of vacuum energy to the dark energy (DE) epoch when the corresponding strongly coupled gauge theory is well known; it is QCD characterized by a single

Published by the American Physical Society under the terms of the Creative Commons Attribution 4.0 International license. Further distribution of this work must maintain attribution to the author(s) and the published article's title, journal citation, and DOI. Funded by SCOAP ${ }^{3}$. dimensional parameter, $\Lambda_{\mathrm{QCD}} \sim 0.1 \mathrm{GeV}$. A similar anomalous coupling (which was the source of the reheating in the holonomy inflation in Ref. [1] when the vacuum energy is transferred to the massless gauge fields) generates the cosmological magnetic helical configurations with an enormous correlation length reaching the size of the entire visible Universe during the present $\mathrm{DE}$ epoch. The focus of the present work is an analysis of the generation mechanism of such a long-ranged magnetic field.

Before we proceed with the outline of this work, we make a few remarks on conventional approaches to study the cosmological magnetic field. We refer to the classical review papers [8,9] and a more recent review [10] for details and references. It is normally assumed that magnetic fields in astronomical structures of different sizes, from stars $R \sim 10^{11} \mathrm{~cm}$ to galaxy clusters $R \sim 10^{24} \mathrm{~cm}$ are produced by the amplification of preexisting weaker "seed" magnetic fields via different types of dynamo. Two broad classes of models for the origin of the seed fields are discussed: (i) A primordial magnetic field (seeds) is produced during different dramatic events in the evolution of the Universe such as inflation, electroweak phase transition, and QCD transition, i.e., during the epochs preceding structure formation; (ii) the process of generation of the seed magnetic fields accompanies the gravitational collapse leading to structure formation. We shall not comment on many problems related to this conventional picture referring to the reviews in Refs. [8-10].

The unorthodox mechanism we are advocating in the present work is drastically distinct from previous conventional approaches. Essentially, the magnetic field in our framework is generated with an enormous scale from the 
moment when it was born, as the source of its energy is the DE occupying the entire Universe. Therefore, there is no need for amplification nor for different types of inverse cascades, as the correlation length of the produced field is already characterized by the largest possible scale. The intensity of this correlated magnetic field is estimated on the level of $B \sim 10^{-10} \mathrm{G}$, and the intensity of the field $B^{2}$ is proportional to the DE density $\rho_{\mathrm{DE}} \approx\left(2.3 \times 10^{-3} \mathrm{eV}\right)^{4}$ with a calculable (in principle) coefficient.

This intensity is very close to the upper limit but not ruled out. In fact, such fields can be studied by future ultrahigh-energy cosmic ray (UHECR) telescopes; see Fig. 14 in Ref. [10].

Our presentation is organized as follows. In Sec. II, we overview the basic ideas and results on the nature of vacuum energy from Ref. [1]. The nature of the DE plays a crucial role in our framework, as it is the source of the cosmic magnetic field, which is the main subject of the present work. Therefore, we overview the basic ideas of Ref. [1] in the context of the present work in great detail for the benefit of the reader. In Sec. III, we explain how the DE couples to the electromagnetic (EM) field through the chiral anomaly. Precisely, this coupling is responsible for the generation of the long-ranged magnetic field, which is the subject of Sec. IV, where we estimate its intensity. We conclude in Sec. V with a few comments on future development and possible observational tests which may support or rule out this new paradigm when DE and the cosmic magnetic field represent two sides of the same coin and are produced at the same epoch.

\section{TOPOLOGY AS THE SOURCE OF THE GRAVITATING VACUUM ENERGY}

The goal here is to overview the basic ideas advocated in Ref. [1]; see also a number of precursor references therein.

In the approach of Ref. [1], the vacuum energy entering the Friedmann equation is defined as $\Delta \rho \equiv \rho_{\mathrm{FRW}}-\rho_{\text {Mink }}$. This definition for vacuum energy for the first time was advocated in 1967 by Zeldovich [11], who argued that $\rho_{\mathrm{vac}}=\Delta \rho \sim G m_{p}^{6}$ must be proportional to the gravitational constant with $m_{p}$ being the proton's mass. Later on, such a definition for the relevant energy $\Delta \rho \equiv \rho_{\text {FRW }}-\rho_{\text {flat }}$ which enters the Einstein equations has been advocated from different perspectives in a number of papers written by researchers from different fields, including particle physics, cosmology, and condensed matter physics. This subtraction prescription is consistent with the conventional subtraction procedure of the divergent ultralocal bare cosmological constant, because in the infinitely large flat spacetime the corresponding contribution is proportional to the $\delta^{4}(x)$ function as explained in Ref. [1]. At the same time, the nontrivial correction to $\Delta \rho$ as discussed below is a nonlocal function of the geometry and cannot be renormalized by any UV counterterms.
In the present work, we consider the geometry $\mathbb{R}^{3} \times \mathbb{S}^{1}$ instead of Friedmann-Robertson-Walker (FRW) geometry to simplify the arguments. The key element in this framework is the presence of a dimensional parameter $\mathcal{T}^{-1}$ which plays the role of the Hubble constant $H$ in FRW geometry which distinguishes FRW geometry from flat infinite spacetime geometry. In other words, we have a dimensional parameter $\mathcal{T}$ which is assumed to be of the order of $\sim H^{-1}$ and which parametrizes the difference between nontrivial and trivial (flat infinite spacetime $\mathbb{R}^{4}$ ) geometries. In computations [1], parameter $\mathcal{T}$ is the proper length of the $\mathbb{S}^{1}$ period. As we already mentioned, this prescription (when $\Delta \rho \equiv\left[\rho_{\text {FRW }}-\rho_{\text {flat }}\right]$ is identified with physical energy, similar to the Casimir effect) is consistent with the Einstein equations when the vacuum energy approaches zero, $\Delta \rho \rightarrow 0$ for the flat spacetime which itself may be considered as a limiting case with $\mathcal{T} \rightarrow \infty$.

The key element of the framework [1] is that the vacuum energy receives the linear correction $\mathcal{T}^{-1}$ at large $\mathcal{T}$ in contrast with naively expected quadratic corrections $\mathcal{T}^{-2}$ such that the vacuum energy entering the Friedmann equation assumes the form

$\rho_{\mathrm{DE}} \equiv\left(E_{\mathrm{vac}}\left[\mathbb{R}^{3} \times \mathbb{S}^{1}\right]-E_{\mathrm{vac}}\left[\mathbb{R}^{3} \times \mathbb{R}^{1}\right]\right)=\Lambda_{\mathrm{QCD}}^{3} \frac{\bar{c}_{\mathcal{T}}}{\mathcal{T}}$,

where the vacuum energy can be represented as follows:

$$
\begin{aligned}
E_{\mathrm{vac}}\left[\mathbb{R}^{3} \times \mathbb{S}^{1}\right] & \simeq-\frac{32 \pi^{2}}{g^{4}} \Lambda_{\mathrm{QCD}}^{4}\left(1-\frac{c_{\mathcal{T}}}{\mathcal{T} \Lambda_{\mathrm{QCD}}}\right) \\
& \simeq-\frac{32 \pi^{2}}{g^{4}} \Lambda_{\mathrm{QCD}}^{4}+\Lambda_{\mathrm{QCD}}^{3} \frac{\bar{c}_{\mathcal{T}}}{\mathcal{T}}+\mathcal{O}\left(\frac{1}{\mathcal{T}^{2}}\right)
\end{aligned}
$$

In this expression, we redefined $\bar{c}_{\mathcal{T}} \equiv \frac{32 \pi^{2}}{g^{4}} c_{\mathcal{T}}$. The coefficient $c_{\mathcal{T}} \sim 1$ is, in principle, a calculable parameter, ${ }^{1}$ expected to be of the order of one. The linear dependence $\mathcal{T}^{-1}$ of the relevant portion of the vacuum energy (1) on external parameter $\mathcal{T}^{-1} \sim H$ suggests that $\rho_{\mathrm{DE}}$ numerically is very close to the observed value today, i.e.,

$$
\rho_{\mathrm{DE}} \simeq \Lambda_{\mathrm{QCD}}^{3} \frac{\bar{c}_{\mathcal{T}}}{\mathcal{T}} \sim \Lambda_{\mathrm{QCD}}^{3} H \sim\left(10^{-3} \mathrm{eV}\right)^{4}
$$

One should also mention that this numerical coincidence in estimate (3) was the main motivation to advocate the proposal $[13,14]$ that the driving force for dark energy is a nontrivial dynamics of the topological sectors in strongly coupled QCD (admittedly, without much deep understanding behind the formula at that time).

A few important comments regarding formulas (1) and (2) are in order.

\footnotetext{
${ }^{1}$ It can be, in principle, computed in strongly coupled QCD using the lattice Monte Carlo simulations, similar to studies [12].
} 
(1) All computations leading to (2) are performed in the Euclidean spacetime where the relevant gauge configurations describing the tunneling processes are defined. Using this technique, one can compute the energy density $\rho$ and the pressure $P$ in Euclidean space. As usual, we assume that there is an analytical continuation to Lorentizan spacetime where the physical energy density has the same form. In our context, it means that the parameters $P$ and $\rho$ and equation of state (EOS) as given by (7) below are interpreted as the corresponding parameters in physical Lorentizan spacetime.

(2) The same arguments also suggest that the parameter $\mathcal{T}$ entering (2) is a constant parameter of the system [not to be confused with observed Hubble $H_{\text {obs }}(t)$, which is time dependent in a FRW universe]. The cosmological evolution in the Lorentizan spacetime is determined by the analytic continuation as discussed in Ref. [1].

(3) What is the interpretation of the parameter $\mathcal{T}$ in physical Lorentizan spacetime? In the system with a Euclidean signature, the parameter $\mathcal{T}$ is determined by the size of $\mathbb{S}^{1}$, which normally can be interpreted as the inverse temperature of the system in Lorentizan spacetime. We think it is a proper interpretation even though there are not any thermodynamical processes which are occurring and characterized by extremely low temperature $\mathcal{T}^{-1} \sim H \sim 10^{-33} \mathrm{eV}$.

(4) The vacuum energy $E_{\text {vac }}$ is defined in a conventional way in terms of the path integral. It has a "nondispersive" nature, which implies that the corresponding vacuum energy cannot be expressed in terms of conventional propagating degrees of freedom (absorptive part) using the dispersion relations to compute the dispersive part. Furthermore, all effects represented by Eq. (2) are obviously nonanalytical in coupling constant $\sim \exp \left(-1 / g^{2}\right)$ and cannot be seen in the perturbation theory. ${ }^{2}$ Nonanalytical structure emerging in Eq. (2) can be easily understood without precise computations. Indeed, $\Lambda_{\mathrm{QCD}}$ in this formula appears as a result of tunneling events which are always proportional to $\Lambda_{\mathrm{QCD}}^{4} \sim \exp \left(-S_{\mathrm{cl}}\right) \sim$ $\exp \left(-1 / g^{2}\right)$, while four zero modes which accompany every magnetic monopole constituent (see item 5 below) of the classical caloron solution with nontrivial holonomy produce the factor $\sim\left[\sqrt{S_{\mathrm{cl}}}\right]^{4} \sim g^{-4}$; see Ref. [7] for the details and references.

\footnotetext{
${ }^{2}$ This nondispersive nature of the vacuum energy is well known to the QCD community: It appears in the computation of topological susceptibility (which is expressed as the second derivative of the vacuum energy with respect to $\theta$ ). The corresponding nondispersive contact term was postulated by Witten in Ref. [15], while the same term with a "wrong sign" in the correlation function was saturated by the Veneziano ghost in Refs. [16,17]; see Appendix A1 in Ref. [1] for references and details.
}

(5) One can view the relevant topological Euclidean configurations which saturate (2) as the $3 \mathrm{~d}$ magnetic monopoles wrapping around the $\mathbb{S}^{1}$ direction. These configurations are characterized by nonvanishing holonomy, which eventually generates the linear (rather than quadratic) correction $\sim 1 / \mathcal{T}$ to the vacuum energy density. For the specific geometry (leading to the de Sitter behavior) considered in Ref. [1], the parameters $\mathcal{T}$ and $H$ are related by $\mathcal{T} \simeq \pi / H$ such that $\Delta \rho \sim H$ when the Hubble parameter is a constant. ${ }^{3}$

(6) In the cosmological context, such configurations are highly unusual objects: They obviously describe the nonlocal physics, as the holonomy is a nonlocal object. Indeed, the holonomy defines the dynamics along the entire history of evolution of the system. This entire gauge configuration is a mere saddle point in the Euclidean path integral computation, which describes the instantaneous tunneling event rather than the propagation of a physical degree of freedom.

(7) The generation of the nondispersive energy $E_{\mathrm{vac}}$ is a highly nonlocal effect, as it is saturated by the gauge configurations with nontrivial holonomy. Precisely, this feature of nonlocality implies that the relevant energy $\Delta \rho$ which enters the Friedmann equation (3) cannot be expressed in terms of a gradient expansion in any effective local field theory.

(8) The basic idea of the framework [1] on the dynamical generation of vacuum energy leading to de Sitter behavior is that there is a linear correction (with respect to the inverse size of the system) to the energy

$$
\frac{E_{\mathrm{vac}}\left[\mathbb{R}^{3} \times \mathbb{S}^{1}\right]}{E_{\mathrm{vac}}\left[\mathbb{R}^{3} \times \mathbb{R}^{1}\right]} \simeq\left(1-\frac{c_{\mathcal{T}}}{\mathcal{T} \Lambda_{\mathrm{QCD}}}\right)
$$

This correction $\sim \mathcal{T}^{-1}$ is generated in spite of the fact that the system has a gap $\Lambda_{\mathrm{QCD}}$ which naively implies that the system must not be sensitive to the size $\mathcal{T}$ of the system at all. We already mentioned that the correction $\mathcal{T}^{-1}$ is nevertheless generated because the vacuum energy (1) and (3) has a nondispersive nature, not associated with any propagating massive degrees of freedom, but rather is related to instantaneous tunneling events (expressed in terms of the Veneziano ghost, mentioned in footnote 2, as the presence of the topologically protected pole). Explicit computations in hyperbolic space $\mathbb{S}^{1} \times \mathbb{M}^{3}$ [7] and a simplified

\footnotetext{
${ }^{3}$ A nonzero holonomy for the vacuum configurations saturating the vacuum energy represents a technical explanation for why the conventional argument [that the correction in (2) must be quadratic in $H^{2}$ in a gravitational background rather than linear in $H]$ fails. The point is that the holonomy is an independent gaugeinvariant nonlocal characteristic of the system, similar to the Polyakov line, which cannot be expressed in terms of the local curvature $R$, which is indeed quadratic in $H$ as $R \sim H^{2}$. Explicit computations in hyperbolic space support this claim; see item 8 .
} 
“deformed QCD" model [18], along with the lattice simulations [12], support this claim.

What is an intuitive way to understand the effect? Imagine that we study the Aharonov-Casher effect. We insert an external charge into a superconductor when the electric field $E$ is screened, i.e., $E \sim Q \exp (-r / \lambda)$ with $\lambda$ being the penetration depth. Nevertheless, a neutral magnetic fluxon will be still sensitive to an inserted external charge $Q$ at arbitrary large distances in spite of the screening of the physical field. This genuine quantum effect is purely topological and nonlocal in nature and can be explained in terms of the dynamics of the gauge sectors which are responsible for the long-range dynamics. Imagine now that we study the same effect but in a time-dependent background. The corresponding topological sectors which saturate the vacuum energy will be modified due to the external background. However, this modification cannot be described in terms of any local dynamical fields, as there are not any propagating longrange fields in the system since the physical electric field is screened. The effect is obviously nonlocal in nature, as the Aharonov-Casher effect itself is a nonlocal phenomenon, and cannot be expressed in terms of the local operator $F_{\mu \nu}$ but rather is expressed in terms of the gauge-invariant, but nonlocal operator, the holonomy $\sim \exp \left(i Q \oint A_{\mu} d x^{\mu}\right)$.

We conclude this short overview on the generation of dynamical vacuum energy (as a result of the dynamics of the topological sectors) with a comment that this type of energy behaves in all respects as a cosmological constant if anomalous coupling with other gauge fields is switched off. Indeed, one can use the conventional thermodynamical relation

$$
d F=T d S-P d V, \quad P=-\left.\frac{\partial F}{\partial V}\right|_{S}
$$

to convince yourself that the correction $\sim \mathcal{T}^{-1}$ does not modify the equation of state. In fact, it behaves exactly in the same way as the cosmological constant does, i.e.,

$$
\begin{aligned}
& P=-\frac{\partial F}{\partial V}=+\frac{32 \pi^{2}}{g^{4}} \Lambda_{\mathrm{QCD}}^{4}\left(1-\frac{c_{\mathcal{T}}}{\mathcal{T} \Lambda_{\mathrm{QCD}}}\right), \\
& \rho=\frac{F}{V}=-\frac{32 \pi^{2}}{g^{4}} \Lambda_{\mathrm{QCD}}^{4}\left(1-\frac{c_{\mathcal{T}}}{\mathcal{T} \Lambda_{\mathrm{QCD}}}\right) .
\end{aligned}
$$

Equation (6) implies that the corresponding equation of state assumes the form

$w=\frac{\Delta P}{\Delta \rho}=-1, \quad \mathrm{a}(t) \sim \exp (H t), \quad H \sim \mathcal{T}^{-1}$,

where $\Delta P$ and $\Delta \rho$ are defined by subtracting the constant value computed in an infinitely large flat spacetime, as explained above and expressed by (1) and (3).
The regime described by (7) would be the final destination of our Universe if the interaction of the QCD gauge configurations (saturating the vacuum energy) with massless EM photons were always switched off. When the coupling of the QCD vacuum fields with the EM field is switched back on, the end of de Sitter behavior is triggered precisely by this interaction, which itself is unambiguously fixed by the triangle anomaly as we discuss in Sec. III.

The corresponding physics of the energy transfer from the vacuum energy given by (1) and (3) to the cosmic magnetic energy is very similar in all respects to the physics of the reheating epoch at the end of inflation when the vacuum energy is transferred to the light gauge SM fields as discussed in Ref. [1]. The technical (very challenging) problems which need to be resolved to address these questions are also very similar in spirit, as we discuss in next section.

\section{COUPLING OF THE VACUUM ENERGY TO PHOTONS}

This section is separated in two parts. In Sec. III A, we explain the formal procedure (based on the Euclidean path integral formulation) which, in principle, allows one to compute the desired rate and other characteristics of the energy transfer. While the corresponding procedure is well defined, it is not technically feasible yet. Therefore, in Sec. III B, we introduce an alternative technique in terms of the auxiliary topological auxiliary fields to attack the problem.

\section{A. Formulation of the problem in terms of the tunneling transitions}

The vacuum energy (1) and (3) in our framework is expressed in terms of the tunneling transitions, which are normally computed in terms of the Euclidean path integral, and the corresponding (Euclidean) field configurations, which describe the interpolation between distinct topologically $|k\rangle$ sectors. In conventional quantum field theory computations, the corresponding procedure selects a specific superposition of the $|k\rangle$ states which generates the $|\theta\rangle$ state with energy $E_{\mathrm{vac}}(\theta)$. In the context of DE, when the background assumes a nontrivial FRW geometry (in contrast with the conventional case described by $\mathbb{R}^{4}$ ), the corresponding computations become profoundly more complicated, though the corresponding procedure is well defined in principle:

(1) One should describe the relevant Euclidean configurations satisfying the proper boundary conditions for a nontrivial geometry (similar to calorons with nontrivial holonomy, reviewed in Appendix A2 in Ref. [1]) represented by the parameter $H \sim \mathcal{T}^{-1}$.

(2) One should compute the corresponding path integral which includes all possible positions and orientations of the relevant gauge configurations interpolating between different topological $|k\rangle$ sectors and physically describing the tunneling transitions between them. 
(3) The corresponding computations for the vacuum energy $\rho$ and pressure $P$ must be done with all massless fields which couple to QCD. In our case, the only massless particles to be considered are the photons, as the production of all massive particles is exponentially suppressed. Precisely, this coupling of the QCD gauge configurations with an EM field is responsible for transferring the vacuum energy to the magnetic energy.

(4) As the last step, one should subtract the corresponding expression (computed on $\mathbb{R}^{4}$ ) as explained in Sec. II. Precisely, this remaining portion of the vacuum energy is interpreted as the relevant energy which enters the Friedmann equation and which cannot be removed by any subtraction procedure and cannot be renormalized by any UV counterterms. The corresponding formulas for $\Delta \rho$ and $\Delta P$ will depend, in general, on properties of the manifold (parametrized by $H$ ), the relevant coupling constant $\alpha$ with an EM field, and the environment where the magnetic field is generated. This procedure will unambiguously predict the magnetic energy of the produced field along with its basic features (such as the correlation length, helical features, etc.).

While these steps are well defined in principle, it is not feasible to perform the corresponding computations, because even the first step in this direction, finding the relevant Euclidean configurations satisfying the proper boundary conditions for a nontrivial geometry, is yet unknown. Nevertheless, this procedure, in principle, shows that the de Sitter behavior (7) in this framework emerges without any local field $\Phi(x)$ as explained in Sec. II, because the physics leading to (7) is not associated with any scalar fields but related to the tunneling events. This procedure, in principle, also shows how the vacuum may transfer its energy to the magnetic field in a time-dependent background.

In many respects, this energy transfer is very similar to the so-called dynamical Casimir effect (DCE) when the photons are radiated from the vacuum in a time-dependent background. The difference with the conventional DCE is that the photons are emitted in our case not from conventional virtual fluctuating particles which always present in the system. The key difference with the DCE is that the photons in our system are emitted from vacuum configurations which describe the tunneling processes between different topological sectors $|k\rangle$.

This difference (in comparison with the DCE) in the nature of emission explicitly displays a hard challenging technical problem in the computation of the corresponding emission rate. Indeed, our topological configurations interpolating between different topological sectors $|k\rangle$ are formulated in terms of the Euclidean path integral, while the emission of real particles on a mass shell represents an inherent Minkowski process. At the present time, the conventional technical tools developed for Euclidean vs Minkowski descriptions are very different and designed for different purposes and different problems. For example, conventional lattice QCD Monte Carlo simulations are not designed to compute physical processes such as on-shell scattering amplitudes but perfectly adapted to compute the Euclidean correlation functions such as topological susceptibility, which assumes a nonzero value exclusively due to the tunneling events between different topological sectors.

\section{B. Formulation of the problem in terms of the auxiliary topological fields}

Fortunately, the key ingredients which are relevant for our future studies can be understood in an alternative way, in terms of the auxiliary topological nonpropagating fields $b(x, H)$ which effectively describe the relevant infrared (IR) physics representing the key elements of steps 1-4 highlighted in Sec. III A. Parameter $H$ here represents the deviation of the manifold under consideration (for example, $1 / \mathcal{T}$ ) from trivial $\mathbb{R}^{4}$.

The basic idea is to construct the effective Lagrangian for the auxiliary topological field $b(x, H)$ using the Euclidean conventional formulation. As the next step, one can utilize the standard formulas to rewrite the corresponding action in Minkowski spacetime. Finally, one can study the emission of real particles and generation of a real magnetic field using the obtained effective Lagrangian written in Minkowski space. This procedure effectively resolves the fundamental technical problem formulated at the end of Sec. III A and originated from the differences in descriptions in Euclidean vs Minkowski spacetimes.

The formal technique we are about to overview is widely used in the particle physics and condensed matter communities. We refer to Appendix B in Ref. [1] for the highlights of the main ideas and results of this approach within the context of the present work. In particular, this approach is extremely useful in the description of the topologically ordered phases when the IR physics is formulated in terms of the topological Chern-Simons(CS-) like Lagrangian. One should emphasize that the corresponding physics, such as the calculation of the braiding phases between quasiparticles, computation of the degeneracy, etc., can be computed (and, in fact, originally had been computed) without a Chern-Simons Lagrangian and without auxiliary fields. Nevertheless, the discussions of the IR physics in terms of CS-like effective action is proven to be very useful, beautiful, and beneficial. In our case, it is not simply a matter of convenience, but in fact a matter of necessity, because we cannot proceed with explicit computations along the lines of $1-4$ as explained in Sec. III A.

In the context of the present work, the auxiliary topological nonpropagating field $b(x, H)$ is introduced in the conventional way as a Lagrange multiplier in the course of inserting the corresponding $\delta$ functional into the path integral which effectively constrains the relevant degrees of freedom; see 
Appendix B in Ref. [1] for references and technical details. ${ }^{4}$ The only information we need in what follows is that auxiliary field $b(x, H)$ should be thought of as the source of the topological fluctuations, similar to the axion field, because it enters the effective Lagrangian precisely in the same way as the $\theta$ parameter enters the fundamental Lagrangian. This claim is explained in Appendix B in Ref. [1] and is based on an analysis of the exact anomalous Ward identities. In many respects, the coupling of the $b(x, H)$ field to the gauge fields is unambiguously determined, similar to the unique coupling of the $\eta^{\prime}$ field to the gluons, photons, and gauge bosons in QCD. Because we know exactly how the $\theta$ parameter couples to electromagnetic $(E \& M)$ fields, we can reconstruct exactly the coupling of the auxiliary topological $b(x, H)$ field with $F_{\mu \nu}$ fields.

As a consequence of this fundamental feature, the topological auxiliary $b(x, H)$ field is, in fact, an angular topological variable, and it has the same $2 \pi$ periodic properties as the original $\theta$ parameter. ${ }^{5}$ In other words, the desired coupling of the $b(x, H)$ field with $F_{\mu \nu}$ photons is

$$
\mathcal{L}_{b \gamma \gamma}(x)=\frac{\alpha}{4 \pi} N \frac{\sum_{i} Q_{i}^{2}}{N_{f}}[\theta+b(x, H)] \cdot F_{\mu \nu} \tilde{F}^{\mu \nu}(x),
$$

where $\alpha$ is the fine-structure constant, $Q_{i}$ are the electric charges of $N_{f}$ light quarks, and $N=3$ is the number of colors of the strongly coupled QCD, and everything is written already in the Minkowski metric. As we already mentioned, the coupling (8) is unambiguously fixed, because the auxiliary $b(x, H)$ field always accompanies the $\theta$ parameter in the specific combination $[\theta+b(x, H)]$ and describes the anomalous interaction of the topological auxiliary $b(x, H)$ field with $E \& M$ photons.

The next question we want to address is as follows: What are the typical fluctuation scales of the auxiliary quantum $b(x, H)$ field? The answer is quite obvious: The typical fluctuations are of the order of $\Lambda_{\mathrm{QCD}}$, as the $b(x, H)$ effectively describes the tunneling events and, in particular, saturates the topological susceptibility (which can be explicitly computed in weakly coupled deformed QCD

\footnotetext{
${ }^{4}$ The computations have been performed in a simplified version of QCD, the so-called weakly coupled deformed QCD model [19] which preserves all relevant features of the strongly coupled QCD such as confinement, nontrivial $\theta$ dependence, generation of the nondispersive vacuum energy, etc., [20]. The corresponding results have been reproduced in Ref. [21] using the technique of the auxiliary topological fields $b(x)$ exploited in the present work. It is expected that a similar description in terms of the auxiliary topological field also holds in strongly coupled QCD. In fact, the Veneziano ghost postulated in Refs. [16,17] can be identified with the auxiliary topological fields [21].

${ }^{5}$ As is known, the $\theta$ parameter can be promoted to the dynamical axion field $\theta(x)$ by adding the canonical kinetic term $\left[\partial_{\mu} \theta(x)\right]^{2}$ to the effective Lagrangian. The difference of the $b(x, H)$ field with the dynamical axion $\theta(x)$ field is that the auxiliary topological field $b(x, H)$ does not have a canonical axion kinetic term.
}

as studied in Ref. [21], where all computations are under complete theoretical control).

What happens when the same system is defined on a nontrivial manifold characterized by some dimensional parameters such as $H \sim \mathcal{T}^{-1} \ll \Lambda_{\mathrm{QCD}}$ ? In this case, the field $b(x, H)$ will continue to fluctuate with typical frequencies $\Lambda_{\mathrm{QCD}}$. However, the relevant correlation functions should demonstrate the emergence of linear corrections with respect to these small parameters $\sim \mathcal{T}^{-1}$. In particular, the topological susceptibility (expressed as the second derivative of the vacuum energy with respect to $\theta$ ) should be of the order of $\Lambda_{\mathrm{QCD}}^{4}$ with corrections of the order of $\left(\Lambda_{\mathrm{QCD}} \mathcal{T}\right)^{-1}$ as expressions (1) and (2) suggest.

It is useful to treat $\dot{b}(x, H)$ as the axial chemical potential, ${ }^{6}$ i.e.,

$$
\mu_{5} \equiv\langle\dot{b}(x, H)\rangle,
$$

which can be easily understood by performing the $U(1)_{A}$ chiral time-dependent transformation in the path integral to rotate away the coupling (8). The corresponding interaction reappears in the form of a singlet nonvanishing axial chemical potential $\mu_{5}$ for light $N_{f}$ flavors as stated in (9).

A few comments are in order. In formula (9), we use notation for the expectation value $\langle\dot{b}(x, H)\rangle$ to emphasize that we treat $b(x, H)$ entering (8) as the external parameter, ignoring a complicated quantum dynamics of the $b(x, H)$ field itself (which would require us to proceed with steps 14 as formulated in Sec. III A). In what follows, we also neglect the backreaction of the $F_{\mu \nu}$ field on $b(x, H)$. In other words, we approximate the dynamics of $b(x, H)$ by taking its expectation value $\langle\dot{b}(x, H)\rangle$ and treating it as an (almost constant) external thermodynamical parameter of the system. One should emphasize that $\mu_{5}$ is not a genuine thermodynamical parameter. Furthermore, $\mu_{5}$ does not satisfy any classical equation of motion, as there is not a canonical kinetic term in the Lagrangian for the $b(x, H)$ field itself. Instead, the $b(x, H)$ field was introduced as a Lagrange multiplier to account for the complicated dynamics of the tunneling events.

Our next comment is related to the estimation of the expectation value $\langle\dot{b}(x, H)\rangle$. As we discussed in Sec. II, the dimensional parameters entering our framework must be computed by subtracting the corresponding expectation values computed on $\mathbb{R}^{4}$. This procedure unambiguously implies $^{7}$ that $\langle\dot{b}(x, H)\rangle \sim H$, as it must vanish at $H=0$ and it must be linear in $H$ as discussed in Sec. II.

\footnotetext{
${ }^{6}$ There is a close analogy with heavy ion physics when a large domain with induced $\theta_{\text {ind }} \neq 0$ can be formed, resulting in the generation of the axial chemical potential $\mu_{5}=\dot{\theta}_{\text {ind }}$ in this $\theta_{\text {ind }}=$ $\mu_{5} t$ domain. This term may produce a number of interesting $P$ odd phenomena; see Ref. [22] for review and references.

${ }^{7}$ For this specific case, $\langle\dot{b}(x, H=0)\rangle=0$. Therefore, the subtraction in this case is a triviality.
} 
Therefore, our problem is now reduced to the study of the magnetic field generation determined by coupling (8) with a source which can be parametrized as follows:

$$
\mu_{5} \equiv\langle\dot{b}(x, H)\rangle=c_{1} H,
$$

where numerical coefficient $c_{1} \sim 1$ is of the order of one, similar to $c_{\mathcal{T}}$ from Eq. (2), and it can be, in principle, computed from first principles by following steps 1-4 as highlighted in Sec. III A.

One should also remark here that many other terms may enter the right-hand side (rhs) in Eq. (10), depending on the geometry. For example, if one considers another geometry with extra $\mathbb{S}_{z}^{1}$ along the $z$ direction, one could expect linear corrections proportional to $\sim c_{z} \mathcal{T}_{z}^{-1}$ similar to $\mathcal{T}^{-1} \sim H$ entering (10). One should also expect the curvature contribution $c_{R} R \sim H_{\mathrm{obs}}^{2}$ representing the conventional quadratic correction; see footnote 3 with a comment. In other words, any deviation from $\mathbb{R}^{4}$, in general, contributes to the rhs in Eq. (10). However, to simplify our analysis in what follows, we limit ourselves with a single parameter $\mathcal{T}^{-1} \sim H$ characterizing the deviation of the geometry with nontrivial holonomy ${ }^{8}$ from the topologically trivial $\mathbb{R}^{4}$. We assume that $c_{1} H$ is the dominating term in Eq. (10).

\section{GENERATION OF THE MAGNETIC FIELD THROUGH THE CHIRAL ANOMALY}

\section{A. Basic equations}

The coupling of the $E \& M$ fields with auxiliary topological field (8) parametrized by (10) generates an additional source term in the Maxwell equations

$$
\vec{\nabla} \times \vec{B}=\sigma \vec{E}+\frac{\alpha}{2 \pi} N \frac{\sum_{i} Q_{i}^{2}}{N_{f}} \cdot\left(\mu_{5} \vec{B}\right),
$$

where $\sigma$ is the conductivity to be estimated below and the term $\sim\langle\vec{\nabla} b(x, H)\rangle \times \vec{E}$ was neglected as a result of the spatial isotropy of the tunneling events. The extra induced nondissipating current $\vec{j} \sim \vec{B}$ has been a very active area of research for many years in a number of different fields, including heavy ion physics (see reviews [22,23]), axion searches (see reviews [24-32]), earlier studies in condensed matter physics $[33,34]$, and more recent studies in condensed matter physics [35] to name just a few.

There are also numerous applications of this anomalous term $\sim \mu_{5} \vec{B}$ to cosmology related to the topic of the present work, and we want to mention just a few papers [36-39] relevant for our future discussions. The drastic difference with most previous studies is that the source (10) in our case is not a dynamical field but, rather, an auxiliary field

\footnotetext{
${ }^{8}$ As we mentioned in Sec. II for a specific geometry studied in Ref. [1] the parameters $H$ and $\mathcal{T}$ are related: $H \simeq \pi / \mathcal{T}$ and describe the de Sitter behavior for constant $H$.
}

accounting for the tunneling transitions in a time-dependent background generating the vacuum dark energy (1) and (3) as discussed in Sec. II. Nevertheless, for our purposes, we can use some technical tools from previous studies treating $\mu_{5}$ as an almost constant thermodynamical parameter.

One should also add that even a constant time-independent $\mu_{5} \neq 0$ is capable to generate the magnetic field in the system. Indeed, the explicit computations in the cosmological context [36] and in heavy ion collision physics [40] support this claim. Our equations (15)-(17) below also suggest that a time-independent $\mu_{5} \neq 0$ generates the magnetic field. Naively, this result may look very suspicious. However, one can easily see that the constant $\mu_{5} \neq 0$ can be treated as the time-dependent phase $\theta \sim \mu_{5} t$; see also footnote 6 . This argument explicitly shows that the time dependence is, in fact, present in the system through the observable phase $\theta(t)$. Therefore, the generation of the magnetic field for time-independent $\mu_{5}$ should not surprise the reader.

With these comments in mind, we consider the following simple ansatz for the magnetic field [38,39]:

$$
\vec{B}=B(t)[\sin (k z), \cos (k z), 0],
$$

while the Bianchi identity $\vec{\nabla} \times \vec{E}=-\frac{\partial \vec{B}}{\partial t}$ implies that the corresponding electric field assumes the form

$$
\vec{E}=-\frac{1}{k} \dot{B}(t)[\sin (k z), \cos (k z), 0]=-\frac{1}{k} \overrightarrow{\dot{B}} .
$$

The configuration (12) is a special case of the force-free field which satisfies

$$
\vec{\nabla} \times \vec{B}=k \vec{B}
$$

see Refs. [38,39] for references and details generalizing the ansatz (12). Substituting (12)-(14) into (11), we arrive to the following equation for $B(t)$ :

$k B(t)=-\frac{\sigma}{k} \dot{B}(t)+\frac{\alpha}{\pi} \bar{c} H B(t), \quad \bar{c} \equiv c_{1} \frac{N \sum_{i} Q_{i}^{2}}{2 N_{f}}$,

where we introduced $\bar{c}$, replacing the previously defined numerical coefficient $c_{1}$ as given by (10).

We are looking for a solution in the form

$$
B(t)=B_{0} \exp (\gamma t)
$$

which returns the following formula for the exponent $\gamma$ :

$$
\gamma=\frac{k}{\sigma}\left[\frac{\alpha}{\pi} \bar{c} H-k\right] .
$$

The exponential growth of the magnetic field occurs for very long waves:

$$
\gamma>0 \Rightarrow k<\frac{\alpha}{\pi} \bar{c} H .
$$


The instability with respect to the generation of the magnetic field $B(t) \sim \exp (\gamma t)$ due to the coupling (8) is a well-known phenomenon and was discussed previously in the literature, including cosmological applications [36-39] and heavy ion collisions [40]. In the context of the inflationary scenario, the same type of coupling could be responsible for the reheating epoch as discussed in Ref. [1].

In the context of the present work, Eqs. (16) and (18) unambiguously imply that the magnetic field will be generated on the enormous scales of the entire visible Universe as a result of anomalous coupling of the DE with the Maxwell field (8). The generation of the magnetic field obviously implies that there will be an energy transfer from the vacuum to the magnetic field as a result of the evolution of the Universe.

One should emphasize that a sample configuration (12) and (13) considered above is oversimplified example. We, of course, do not expect the magnetic field to be uniform running along the $z$ direction through the entire Universe. Instead, we expect the field to be twisted, as it is highly helical (which is normally associated with linking and twisting of magnetic fluxes). Indeed, the magnetic helicity is defined as

$$
\mathcal{H} \equiv \int \vec{A} \cdot \vec{B} d^{3} x .
$$

The time evolution of the magnetic helicity is determined precisely by $\vec{E} \cdot \vec{B}$ entering Eq. (8), i.e.,

$$
\frac{d \mathcal{H}}{d t}=-2 \int \vec{E} \cdot \vec{B} d^{3} x .
$$

For our configuration (12) and (13) considered above, the magnetic helicity per unit volume $\mathcal{H} / V$ is directly related to the magnetic energy density, i.e.,

$$
\frac{\mathcal{H}(t)}{V} \approx \frac{B^{2}(t)}{k} .
$$

Furthermore, the time evolution of both observables is also the same as Eq. (21) states.

One should comment here that a magnetic field with an enormous correlation length is known to be present in our Universe; see the original paper [41] and review [10]. The mechanism suggested in the present work automatically generates fields with such large correlation lengths. On other hand, it is very hard, if at all possible, to generate such an enormous correlation length within conventional approaches; see Ref. [10] for review.

The generation of the magnetic field from $\mu_{5}$ is not a very new idea and was previously discussed in the literature for different systems. Furthermore, it has been known for some time that the generation of the helical magnetic field is normally accompanied by a decrease of $\mu_{5}$, which is the source of the produced field. In particular, such behavior is shown to occur in heavy ion systems [40] and also in the systems relevant for cosmology $[38,39]$.
What is the efficiency of this energy transfer from DE to magnetic energy in our case? What is the typical time scale for this energy transfer? What is the intensity of the magnetic field generated by this mechanism? We have to estimate $\sigma$ and other related parameters in order to address these and many other related questions, which is the topic of the next subsection.

\section{B. Numerical estimates}

This subsection is much more speculative in comparison with our previous discussions in Sec. IVA, which is entirely based on the Maxwell equations in the presence of an additional axion term. Nevertheless, we want to proceed with our speculations here to argue that all conventional cosmological assumptions about the environment lead to estimates for the magnetic field which are perfectly consistent with presently available observations. Future studies as discussed in Ref. [10] are capable to discover these long-ranged fields.

We start with electric conductivity $\sigma$ entering the expression (17) for $\gamma$. It is normally estimated as follows:

$$
\sigma=\frac{4 \pi n_{e} \alpha \tau}{m_{e}}
$$

where $\tau$ is the timescale when a free electron is losing its coherence. This timescale for a low-density environment is normally estimated as a result of the interaction of electrons with cosmic microwave background (CMB) photons through Thomson scattering:

$$
\tau^{-1}=n_{\gamma} \sigma_{T}, \quad \sigma_{T}=\frac{8 \pi \alpha^{2}}{3 m_{e}^{2}},
$$

where in conventional circumstances $n_{\gamma}=n_{\mathrm{CMB}} \sim T^{3}$; see, e.g., Ref. [9]. However, as we estimate below, in our framework the number density of the $E \& M$ configurations characterized by $\vec{B}$ and $\vec{E}$ fields and given by (12) and (13) correspondingly is much higher than $n_{\mathrm{CMB}}$. Precisely, these long-wavelength configurations with very low $k$ as given by (18) will be dominating the electron resistivity in a lowdensity environment.

The estimation for the electron density $n_{e}$ entering (22) strongly depends on the scale under consideration. For example, if residual free electrons (after recombination $p+e \leftrightarrow H+\gamma)$ dominate the physics, their density is estimated as [9]

$$
n_{e} \approx 2 \times 10^{-10}(1+z)^{3} \mathrm{~cm}^{-3} .
$$

At the same time, if one assumes that the intergalactic medium is mostly ionized, then $n_{e}$ is about the average baryon density [10]:

$$
n_{e} \approx \frac{\rho_{B}}{m_{p}} \approx 2 \times 10^{-7}(1+z)^{3} \mathrm{~cm}^{-3},
$$

where $\rho_{B}$ is the baryon density. 
To proceed with our task, we have to estimate $n_{\gamma}$ entering (23). We define the corresponding density $n_{\gamma}$ as follows:

$$
\hbar \omega_{k} \cdot n_{\gamma}(t) \equiv \frac{B^{2}(t)}{2} .
$$

For convenience of the estimates, we also introduce the dimensionless suppression factor $\xi(t)<1$, which relates the magnetic energy density in comparison with the DE density, i.e.,

$$
\frac{B^{2}(t)}{2} \simeq \xi(t) \cdot \rho_{\mathrm{DE}}(t),
$$

where $\rho_{\mathrm{DE}}(t)$ is the source of the magnetic energy and it is defined in our framework by Eqs. (1) and (3). Our goal is to estimate $\xi(t)$ and, therefore, the strength of the magnetic field $B(t)$.

To achieve this goal, we estimate the ratio $k / \sigma$ entering the expression for $\gamma$ in terms of the observable parameters as follows:

$$
\frac{k}{\sigma}=\frac{m_{e} k n_{\gamma} \sigma_{T}}{4 \pi n_{e} \alpha} \simeq \frac{\alpha}{3}\left(\frac{B^{2}}{n_{e} m_{e}}\right) \simeq \xi \frac{2 \alpha}{3}\left(\frac{\rho_{\mathrm{DE}}}{n_{e} m_{e}}\right),
$$

where we used previously defined relations (22), (23), (26), and (27) and for estimates we take $\omega_{k} \approx k$. Numerically, one has

$$
\frac{k}{\sigma} \sim 2 \times 10^{2} \xi\left(\frac{2 \times 10^{-7} \mathrm{~cm}^{3}}{n_{e}}\right) .
$$

This is precisely the dimensionless parameter which enters expression (17) for $\gamma$. It measures, according to (16), the typical timescale (in Hubble units $H^{-1}$ ) when the magnetic field is generated. In other words, the energy transfer from $\mathrm{DE}$ to magnetic energy becomes highly efficient when $\left(\gamma \bar{\tau}_{\text {form }}\right) \sim 1$.

To proceed with the estimates, we need to make one more assumption, which is formulated as follows. It is normally assumed (in strongly coupled systems) that, in order to form a configuration characterized by a typical energy $\omega$, one needs a timescale of the order of $(2 \pi) / \omega$, which is essentially a trivial manifestation of the uncertainty relation. In our (weakly coupled) case, there is an additional fine-structure coupling constant $\alpha /(2 \pi)$ entering (8), which suggests that the timescale $\tau_{\text {form }}$ required to form the magnetic configuration with wavelength $k^{-1}$ from the DE source is $(2 \pi / \alpha)^{2}$ much longer. In addition, the time which is available for the present Universe is $H_{\mathrm{obs}}^{-1}$, which is much shorter than $\omega_{k}^{-1}$ according to (18). This implies that $\gamma \tau_{\text {form }}$ still cannot reach a magnitude of order one at the present time; instead, it assumes only a fraction of its value $\gamma \tau_{\text {form }} \sim\left(\frac{\alpha \bar{c}}{\pi}\right) \gamma \bar{\tau}_{\text {form }}$, because $\frac{\omega_{k}}{H_{\text {obs }}} \sim \frac{\alpha \bar{c}}{\pi}$ at the present time when $H \sim H_{\text {obs. }}$. Collecting all these factors together, we arrive at our final estimate:

$$
\begin{aligned}
\gamma \tau_{\text {form }} & \sim \frac{k}{\sigma}\left[\frac{\alpha}{\pi} \bar{c} H-k\right] \cdot\left(\frac{2 \pi}{\omega_{k}}\right) \cdot\left(\frac{2 \pi}{\alpha}\right)^{2} \\
& \sim 2 \times 10^{2} \xi \cdot\left(\frac{2 \times 10^{-7} \mathrm{~cm}^{3}}{n_{e}}\right) \cdot \frac{(2 \pi)^{3}}{\alpha^{2}} \\
& \approx\left(\frac{\omega_{k}}{H_{\mathrm{obs}}}\right) \sim \frac{\alpha \bar{c}}{\pi}
\end{aligned}
$$

where we approximated $\omega_{k} \sim k$ and used the estimate (29) for $k / \sigma$.

The numerical value for $\xi$ which follows from relation (30) can be written as follows:

$$
\xi \sim 10^{-2} \frac{\alpha^{3} \bar{c}}{(2 \pi)^{4}}\left(\frac{n_{e}}{2 \times 10^{-7} \mathrm{~cm}^{3}}\right) \sim 10^{-12} \bar{c},
$$

which implies that the intensity of the magnetic field at the present time assumes the following value:

$B \sim \sqrt{\xi \rho_{\mathrm{DE}}} \sim 10^{-6} \cdot\left(2.3 \times 10^{-3} \mathrm{eV}\right)^{2} \sim 2.6 \times 10^{-10} \mathrm{G}$,

where we expressed $\mathrm{eV}^{2}$ units in terms of conventional Gauss using the following relation: $1 \mathrm{G} \simeq 2 \times 10^{-2} \mathrm{eV}^{2}$.

This is, of course, an order of magnitude estimate. However, the important point here is not just that a relatively strong magnetic field can be generated by this mechanism. A much more important element of the proposed mechanism is that the source of this field is the vacuum dark energy $\rho_{\mathrm{DE}}$ such that these two (naively unrelated) cosmological puzzles (the nature of the dark energy and magnetic coherent field) are intimately related, because they both originated from the same physics governed by the dynamics of the QCD topological sectors as reviewed in Sec. II.

One may wonder if the generation of the magnetic field at earlier times could produce a larger intensity field in comparison with estimate (32). The answer is "no," and the reason for that is as follows. The wavelength $k$ is determined by Eq. (18), where parameter $H$ is defined in (3). At earlier times, the right-hand side of Eq. (30) will have an additional suppression factor $H / H_{\mathrm{obs}} \ll 1$, as the time formation in physical units is getting shorter for the same frequency $\omega_{k}$. This implies that parameter $\xi$, and, therefore, the intensity of the generated magnetic field, will receive an additional suppression. This argument implies that the strongest field is generated last. It should be contrasted with conventional mechanisms which could produce a very strong field at the moment of formation but become very weak due to the Hubble expansion.

\section{CONCLUSION AND FUTURE DIRECTIONS}

The main claim of this work is that the tunneling transitions in QCD in the expanding Universe will 
generate the coupling (8) due to the chiral anomaly. This interaction unambiguously implies that the Maxwell equations will be modified according to Eq. (11). This additional nondissipating current $\vec{j} \sim \vec{B}$ in the Maxwell system implies that there will be an energy transfer from vacuum $\mathrm{DE}$ to magnetic energy. The correlation length of the produced magnetic field is determined by the DE correlation length as $B^{2} \sim \rho_{\mathrm{DE}}$ in this framework. The intensity of the field generated by this mechanism is estimated on the level of $B \sim 10^{-10} \mathrm{G}$ according to (32).

Can we test some of these ideas in tabletop experiments, at least, as a matter of principle? We want to argue that the ultimate answer is "yes." Therefore, we claim that we are dealing with a real physics phenomenon rather than with a joggling of formal equations (such as insertions of the Lagrange multipliers, introduction of the auxiliary fields, subtractions of the UV counterterms, and other formal elements which may look very suspicious for some readers).

The basic idea for a tabletop experiment goes as follows. The fundamentally new type of energy discussed in Sec. II can be, in principle, studied by measuring some specific corrections to the Casimir vacuum energy in the Maxwell theory as suggested in Refs. [42-46]. This fundamentally new contribution to the Casimir pressure emerges as a result of tunneling processes rather than due to the conventional fluctuations of the propagating photons with two physical transverse polarizations. Therefore, it was coined as the topological Casimir effect. The extra energy computed in Refs. [42-46] is the direct analog of the QCD nondispersive vacuum energy (1) and (2), which is the key player of the present work as it explicitly enters the EOS (7). In fact, an extra contribution to the Casimir pressure emerges in this system as a result of nontrivial holonomy for the Maxwell field. The nontrivial holonomy in the $E \& M$ system is enforced by the nontrivial boundary conditions imposed in Refs. [42-46] and related to the nontrivial mapping $\pi_{1}[U(1)]=\mathbb{Z}$ relevant for the Maxwell Abelian gauge theory.

Furthermore, the emission of real physical photons from the Euclidean vacuum configurations describing the tunneling events in the Abelian Maxwell system (representing the direct analog of the non-Abelian system discussed in Sec. III A) can also be studied in the Maxwell theory as argued in Ref. [45].

In fact, the same obstacle (related to the formulation of the tunneling transitions in terms of the Euclidean path integral, while the emission of real particles on a mass shell represents an inherent Minkowski process) can be also resolved by introducing the auxiliary topological fields in the Maxwell system, similar to the discussions in Sec. III B; see Ref. [45] for the details.

To recapitulate the main point, the long-range magnetic field with an intensity of the order of $B \sim 10^{-10} \mathrm{G}$ can be generated as a result of the variation of the QCD tunneling transition rate in the time-dependent background related to the Universe expansion. The two naively distinct phenomena are, in fact, closely related, as the DE is the source for the magnetic energy in this framework, $B^{2} \sim \rho_{\mathrm{DE}}$. This novel effect can be, in principle, tested in a tabletop experiment and in many respects is similar to the dynamical Casimir effect. What is more important is that such fields (correlated on the enormous scale of the visible Universe) can be studied by future UHECR telescopes; see Fig. 14 in Ref. [10]. We finish this work on this optimistic note.

\section{ACKNOWLEDGMENTS}

This research was supported in part by the Natural Sciences and Engineering Research Council of Canada.

\footnotetext{
${ }^{9} \mathrm{~A}$ similar new type of energy can be, in principle, also studied in the superfluid He-II system which also shows a number of striking similarities with non-Abelian QCD as argued in Ref. [47]. For the superfluid He-II system, the crucial role is played by the vortices which are classified by $\pi_{1}[U(1)]=\mathbb{Z}$ similar to the Abelian quantum fluxes studied in the Maxwell system.
}

[1] A. O. Barvinsky and A. R. Zhitnitsky, Phys. Rev. D 98, 045008 (2018).

[2] A. O. Barvinsky and A. Y. Kamenshchik, J. Cosmol. Astropart. Phys. 09 (2006) 014.

[3] A. O. Barvinsky and A. Y. Kamenshchik, Phys. Rev. D 74, 121502 (2006).

[4] A. O. Barvinsky, Phys. Rev. Lett. 99, 071301 (2007).

[5] A. R. Zhitnitsky, Phys. Rev. D 89, 063529 (2014).

[6] A. R. Zhitnitsky, Phys. Rev. D 90, 043504 (2014).

[7] A. R. Zhitnitsky, Phys. Rev. D 92, 043512 (2015).

[8] P. P. Kronberg, Rep. Prog. Phys. 57, 325 (1994).
[9] D. Grasso and H. R. Rubinstein, Phys. Rep. 348, 163 (2001).

[10] R. Durrer and A. Neronov, Astron. Astrophys. Rev. 21, 62 (2013).

[11] Y. B. Zeldovich, Pis'ma Zh. Eksp. Teor. Fiz. 6, 883 (1967) [JETP Lett. 6, 316 (1967)].

[12] A. Yamamoto, Phys. Rev. D 90, 054510 (2014).

[13] F. R. Urban and A. R. Zhitnitsky, Phys. Lett. B 688, 9 (2010).

[14] F. R. Urban and A. R. Zhitnitsky, Nucl. Phys. B835, 135 (2010). 
[15] E. Witten, Nucl. Phys. B156, 269 (1979).

[16] G. Veneziano, Nucl. Phys. B159, 213 (1979).

[17] P. Di Vecchia and G. Veneziano, Nucl. Phys. B171, 253 (1980).

[18] E. Thomas and A. R. Zhitnitsky, Phys. Rev. D 86, 065029 (2012).

[19] M. Ünsal and L. G. Yaffe, Phys. Rev. D 78, 065035 (2008).

[20] E. Thomas and A. R. Zhitnitsky, Phys. Rev. D 85, 044039 (2012).

[21] A. R. Zhitnitsky, Ann. Phys. (Amsterdam) 336, 462 (2013).

[22] D. E. Kharzeev, Ann. Phys. (Amsterdam) 325, 205 (2010).

[23] D. E. Kharzeev, J. Liao, S. A. Voloshin, and G. Wang, Prog. Part. Nucl. Phys. 88, 1 (2016).

[24] K. van Bibber and L. J. Rosenberg, Phys. Today 59, No. 8, 30 (2006);

[25] S. J. Asztalos, L. J. Rosenberg, K. van Bibber, P. Sikivie, and K. Zioutas, Annu. Rev. Nucl. Part. Sci. 56, 293 (2006).

[26] P. Sikivie, Lect. Notes Phys. 741, 19 (2008).

[27] G. G. Raffelt, Lect. Notes Phys. 741, 51 (2008).

[28] P. Sikivie, Int. J. Mod. Phys. A 25, 554 (2010).

[29] L. J. Rosenberg, Proc. Natl. Acad. Sci. U.S.A. 112, 12278 (2015),

[30] P. W. Graham, I. G. Irastorza, S. K. Lamoreaux, A. Lindner, and K. A. van Bibber, Annu. Rev. Nucl. Part. Sci. 65, 485 (2015).

[31] D. J. E. Marsh, Phys. Rep. 643, 1 (2016).

[32] A. Ringwald, Proc. Sci. NOW2016 (2016) 081.
[33] A. Y. Alekseev, V. V. Cheianov, and J. Frohlich, Phys. Rev. Lett. 81, 3503 (1998).

[34] J. Frohlich and B. Pedrini, arXiv:cond-mat/0201236.

[35] Q. Li, D. E. Kharzeev, C. Zhang, Y. Huang, I. Pletikosić, A. V. Fedorov, R. D. Zhong, J. A. Schneeloch, G. D. Gu, and T. Valla, Nat. Phys. 12, 550 (2016).

[36] S. M. Carroll, G. B. Field, and R. Jackiw, Phys. Rev. D 41, 1231 (1990).

[37] M. Joyce and M. E. Shaposhnikov, Phys. Rev. Lett. 79, 1193 (1997).

[38] A. Boyarsky, J. Frohlich, and O. Ruchayskiy, Phys. Rev. Lett. 108, 031301 (2012).

[39] A. Boyarsky, J. Frohlich, and O. Ruchayskiy, Phys. Rev. D 92, 043004 (2015).

[40] Y. Akamatsu and N. Yamamoto, Phys. Rev. Lett. 111, 052002 (2013).

[41] A. Neronov and I. Vovk, Science 328, 73 (2010).

[42] C. Cao, M. van Caspel, and A. R. Zhitnitsky, Phys. Rev. D 87, 105012 (2013).

[43] A. R. Zhitnitsky, Phys. Rev. D 91, 105027 (2015).

[44] C. Cao, Y. Yao, and A. R. Zhitnitsky, Phys. Rev. D 93, 065049 (2016).

[45] Y. Yao and A. R. Zhitnitsky, Phys. Rev. D 95, 065018 (2017).

[46] C. Cao and A. Zhitnitsky, Phys. Rev. D 96, 015013 (2017).

[47] A. Zhitnitsky, Nucl. Phys. B916, 510 (2017). 